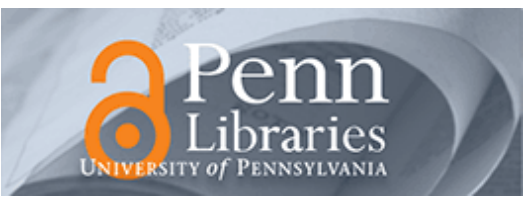

University of Pennsylvania

ScholarlyCommons

June 1996

\title{
Evaluation of Few-View Reconstruction Parameters for Illicit Substance Detection using Fast-Neutron Transmission Spectroscopy
}

\author{
C. L. Fink \\ University of Chicago \\ P. G. Humm \\ University of Chicago \\ Milo Martin \\ University of Pennsylvania, milom@cis.upenn.edu \\ B. J. Micklich \\ University of Chicago \\ Follow this and additional works at: https://repository.upenn.edu/cis_papers
}

\section{Recommended Citation}

C. L. Fink, P. G. Humm, Milo Martin, and B. J. Micklich, "Evaluation of Few-View Reconstruction

Parameters for Illicit Substance Detection using Fast-Neutron Transmission Spectroscopy", . June 1996.

Copyright 1996 IEEE. Reprinted from IEEE Transactions on Nuclear Science, Volume 43, Issue 3, June 1996, pages 1352-1356.

This material is posted here with permission of the IEEE. Such permission of the IEEE does not in any way imply IEEE endorsement of any of the University of Pennsylvania's products or services. Internal or personal use of this material is permitted. However, permission to reprint/republish this material for advertising or promotional purposes or for creating new collective works for resale or redistribution must be obtained from the IEEE by writing to pubs-permissions@ieee.org. By choosing to view this document, you agree to all provisions of the copyright laws protecting it.

NOTE: At the time of publication, the author was affiliated with Argonne National Laboratory. Currently March 2007, he is a faculty member of the Department of Computer and Information Sciences at the University of Pennsylvania.

This paper is posted at ScholarlyCommons. https://repository.upenn.edu/cis_papers/320

For more information, please contact repository@pobox.upenn.edu. 


\title{
Evaluation of Few-View Reconstruction Parameters for Illicit Substance Detection using Fast-Neutron Transmission Spectroscopy
}

\begin{abstract}
We have evaluated the performance of an illicit substance detection system that performs image reconstruction using the Maximum Likelihood algebraic reconstruction algorithm, a few number of projections, and relatively coarse projection and pixel resolution. This evaluation was done using receiver operator curves and simulated data from the fast-neutron transmission spectroscopy system operated in a mode to detect explosives in luggage. The results show that increasing the number of projection angles is more important than increasing the projection resolution, the reconstructed pixel resolution, or the number of iterations in the Maximum Likelihood algorithm. A $100 \%$ detection efficiency with essentially no false positives is possible for a square block of RDX explosive, a projection resolution of $2 \mathrm{~cm}$, a reconstructed pixel size of $2 \times 2 \mathrm{~cm}$, and five projection angles. For rectangular shaped explosives more angles are required to obtain the same system performance.
\end{abstract}

\section{Comments}

Copyright 1996 IEEE. Reprinted from IEEE Transactions on Nuclear Science, Volume 43, Issue 3, June 1996, pages 1352-1356.

This material is posted here with permission of the IEEE. Such permission of the IEEE does not in any way imply IEEE endorsement of any of the University of Pennsylvania's products or services. Internal or personal use of this material is permitted. However, permission to reprint/republish this material for advertising or promotional purposes or for creating new collective works for resale or redistribution must be obtained from the IEEE by writing to pubs-permissions@ieee.org. By choosing to view this document, you agree to all provisions of the copyright laws protecting it.

NOTE: At the time of publication, the author was affiliated with Argonne National Laboratory. Currently March 2007, he is a faculty member of the Department of Computer and Information Sciences at the University of Pennsylvania. 


\title{
Evaluation of Few-View Reconstruction Parameters for Illicit Substance Detection using Fast-Neutron Transmission Spectroscopy
}

\author{
C. L. Fink, P. G. Humm, M. M. Martin, and B. J. Micklich \\ Argonne National Laboratory \\ Argonne, IL 60439
}

\begin{abstract}
We have evaluated the performance of an illicit substance detection system that performs image reconstruction using the Maximum Likelihood algebraic reconstruction algorithm, a few number of projections, and relatively coarse projection and pixel resolution. This evaluation was done using receiver operator curves and simulated data from the fast-neutron transmission spectroscopy system operated in a mode to detect explosives in luggage. The results show that increasing the number of projection angles is more important than increasing the projection resolution, the reconstructed pixel resolution, or the number of iterations in the Maximum Likelihood algorithm. A $100 \%$ detection efficiency with essentially no false positives is possible for a square block of RDX explosive, a projection resolution of $2 \mathrm{~cm}$, a reconstructed pixel size of $2 \times 2 \mathrm{~cm}$, and five projection angles. For rectangular shaped explosives more angles are required to obtain the same system performance.
\end{abstract}

\section{INTRODUCTION}

The Fast-Neutron Transmission Spectroscopy (FNTS) technique is one of several neutron interrogation methods being examined for detection of illicit substances such as explosives and drugs [1-3]. The use of fast neutrons is attractive because, unlike $x$-ray transmission, neutron transmission is more sensitive to the presence of the light elements $\mathrm{H}, \mathrm{C}, \mathrm{N}$, and $\mathrm{O}$, which are the major constituents of explosives and narcotics.

The FNTS technique was first used by Overly [4] to determine compositions of bulk organic materials. The technique uses an accelerator to produce nanosecond pulsed beams of protons or deuterons that strike a target and produce a pulsed beam of neutrons with a continuum of energies. The interrogated material is placed in the flight path between the accelerator target and the neutron detector array, and time-offlight techniques are used to measure the neutron transmission through the sample as a function of neutron energy. Since the neutron total cross section for light elements varies widely in the measured neutron-energy range, it is relatively easy to unfold the elemental projection densities from the measured transmission data [5].

Overlap of elemental densities from different objects along the projection path leads to false negatives (failure to detect explosives/drugs when present) and false positives (the detection of explosives/drugs when not actually present). Thus it will be necessary to use tomographic reconstruction techniques to provide partial separation of objects. These tomographic reconstructions will use only a few projections ( 3 to 7) and use relatively large projection and pixel resolutions $(1-3 \mathrm{~cm})$ because of constraints in inspection time and detector cost.

\section{SYSTEM DESCRIPTION}

A FNTS inspection system requires four distinct steps to arrive at a decision on the presence or absence of an explosive in luggage. These are (1) the unfolding of the elemental projection density information from the transmission data, (2) the tomographic reconstruction of the elemental density distributions within the suitcase, (3) the combining of the elemental density distributions into an explosive signature that maximizes sensitivity to the explosive and minimizes sensitivity to background objects, and (4) the use of an image processing algorithm to separate the explosive signature from background noise. The performance of a luggage inspection system is often characterized by receiver operator characteristic (ROC) curves, which show the relationship between true positives (indication of an explosive in luggage actually containing an explosive) and false positives (indication of an explosive in luggage that does not contain explosives). In this paper we have used the exact projection data with no noise and a relatively simple explosive signature and image processing algorithm to study the effects of varying the tomographic reconstruction parameters on the systems ROC performance. In particular we were interested in the effect on system performance of varying the number of projection angles and system resolution. 


\section{A. Elemental Unfolding}

In these studies we will use the exact projection data as calculated from the actual elemental densities in the slice being interrogated. Previous work using Monte Carlo simulations of the transmission for relatively simple phantoms $[2,6]$ has shown good agreement between calculated and exact projection densities.

\section{B. Tomographic Reconstruction}

Because the number of projection angles will be limited, the FNTS system uses algebraic reconstruction techniques. For this initial evaluation we have used the Maximum Likelihood Method [7] although other reconstruction algorithms are being evaluated. We have limited the number of iterations to between 25 and 50. For this evaluation, we assumed that the suitcase would be examined in a series of square slices with the dimensions of each slice corresponding to a $60-\mathrm{cm}$ square. The projection resolution was varied between 1 and $2 \mathrm{~cm}$ and the reconstructed pixel resolution between 1 and $3 \mathrm{~cm}$. In these studies the explosive shapes have been limited to an $8-\mathrm{cm}$ square or a $32-\mathrm{cm}$ by $2-\mathrm{cm}$ rectangle.

\section{Explosive Signature}

The tomographic reconstruction provides the $\mathrm{H}, \mathrm{C}, \mathrm{N}$, and $\mathrm{O}$ density distribution. These density distributions must be combined to maximize the signature from an explosive and to minimize the background signature from nonexplosive objects. The process is further complicated by the fact that a wide range of explosives must be considered. For this study we use the concept of maximum equivalent explosive signature [7]. Each pixel has a calculated $\mathrm{H}, \mathrm{C}, \mathrm{N}$, and $\mathrm{O}$ density. The equivalent explosive signature for a pixel is obtained by dividing each of these measured elemental densities by the corresponding elemental mass fraction of the explosive of interest. The smallest of the four densities is the equivalent explosive density since the element yielding the smallest value limits the amount of explosive present in the pixel. If there is the possibility of several different types of explosives, we calculate an equivalent explosive density for each explosive and use the largest. Note that effectiveness of this algorithm is enhanced by the high density of explosives $\left(\sim 1.6 \mathrm{~g} / \mathrm{cm}^{3}\right)$. Since we were mainly interested in studying the effect of the reconstruction algorithms, we used a single explosive (RDX) in the system evaluation.

\section{Image Processing}

To detect the presence of explosives, we used a simple binary image processing algorithm in which an explosive is considered present in the image if the area of all pixels greater than some threshold is greater than some specified value. Thus the decision variable in generating the ROC curves is a function of two parameters. Clearly the image detection algorithm could be improved by also including information on the spatial distribution of the pixels above the threshold.

\section{ROC CURVES}

The effects of the reconstruction parameters were evaluated using ROC curves generated by determining the performance of an FNTS system on two sets of 50 randomly generated suitcases. The first set consisted of suitcases with no explosives; the second set consisted of the same suitcases but with an explosive added.

\section{A. Generation of Random Suitcases}

Since there is relatively little data on the actual contents of suitcases and since the actual packing is also unknown, we chose a somewhat mechanistic approach to generate randomly packed suitcases. First, we placed four background objects within the suitcase. The composition of the background objects were randomly selected from a list of materials that were characteristic of low-density materials available in a suitcase. The most obvious example of a background object would be wool or cotton clothing packed to a density of 0.2 $\mathrm{g} / \mathrm{cm}^{3}$. Next, the shape of background object (either rectangular or elliptical), the major and minor axes, the orientation, and the position within the suitcase are randomly selected. To avoid problems with fitting the background objects together, we simply replaced any previous background material with the new background material in places where the background objects overlapped. Similarly any portion of the background object that laid outside the dimensions of the suitcase was discarded.

Once the background objects were placed in the suitcase, 20 ordinary objects were selected from a list of materials and randomly positioned within the slice in the same fashion as the background objects. The only difference with these ordinary objects relative to the background objects was the size distribution. The ordinary objects had sizes from 0 to $30 \%$ of the suitcase dimension while the background objects had sizes from 50 to $100 \%$. The final object placed in a phantom was 
the explosive. The shape of the explosive was fixed but its position and orientation were varied randomly.

Figures 1 and 2 show the $\mathrm{C}, \mathrm{H}, \mathrm{N}$, and $\mathrm{O}$ density distributions for two suitcases generated in this manner. The explosive in these figures corresponds to a $32-\mathrm{cm}$ by $2 \mathrm{~cm}$ rectangle.

The equivalent explosive density after a typical reconstruction using 5 angles and a $2 \times 2 \mathrm{~cm}$ reconstructed pixel resolution is shown in Fig. 3 with and without explosives. These reconstructed images where generated from the projections using the elemental distributions in Figs. 1 and 2.

\section{B. Generation of ROC Curves}

A ROC curve is generated by plotting the percentage of true positives detected versus the corresponding percentage of

C: Exp Trial 1

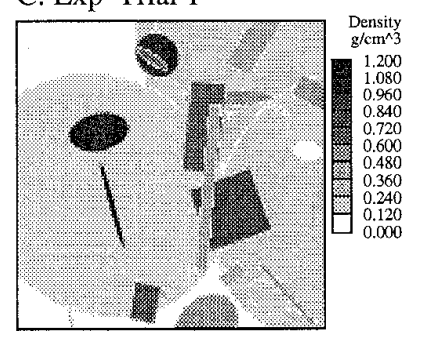

N: Exp Trial 1

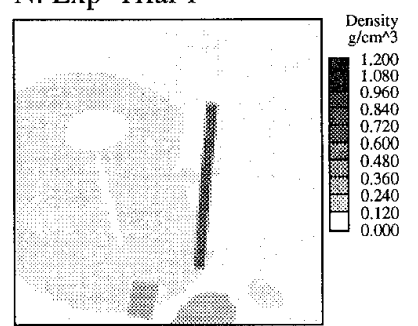

Fig. 1. Example of the elemental density distributions for a randomly packed suitcase (Trial 1) containing an explosive.

false positives as a function of a decision variable. In our case the decision value is a function of both the equivalent explosive density threshold and the number of pixels required to have a density greater than or equal to this threshold. Thus the true-positive percentage corresponds to the percentage of the suitcases containing explosives that had an area greater than or equal to the specified area for the specified threshold. The false-positive percentage corresponds to the number of suitcases without explosives that also had an area greater to or equal to the specified area for the specified threshold.
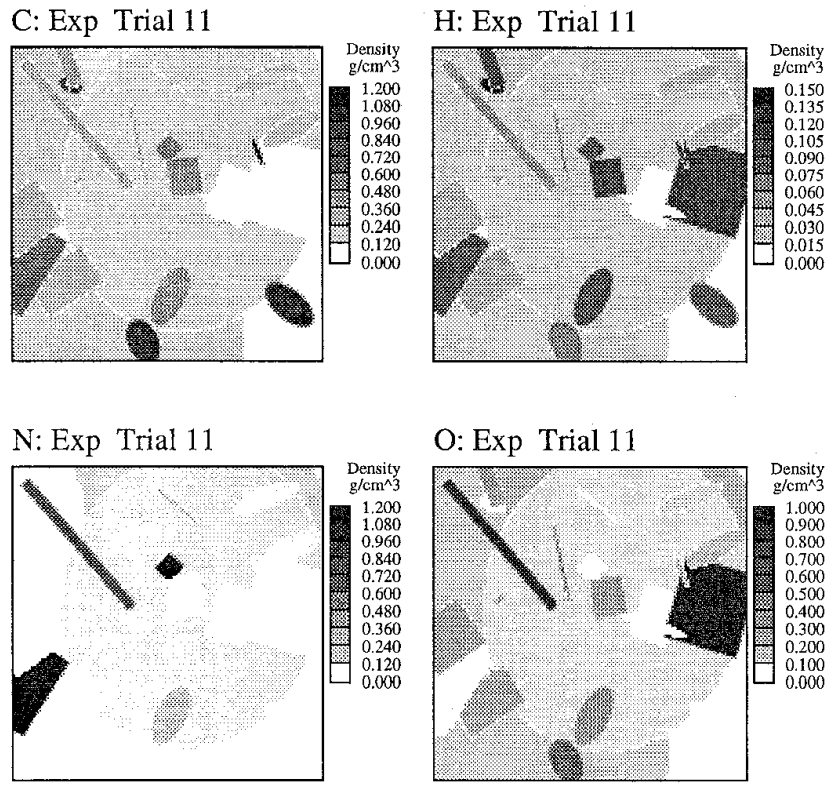

Fig. 2. Example of the elemental density distributions for a randomly packed suitcase (Trial 11) containing an explosive.
EX: NoExp Trial 1

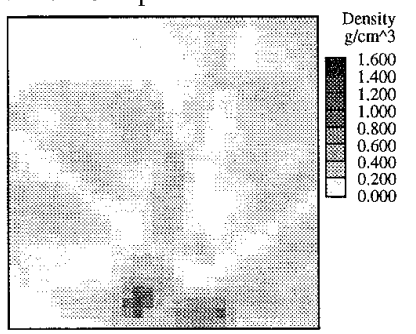

EX: Exp Trial 1

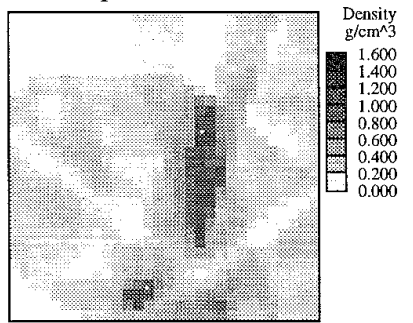

EX: NoExp Trial 11

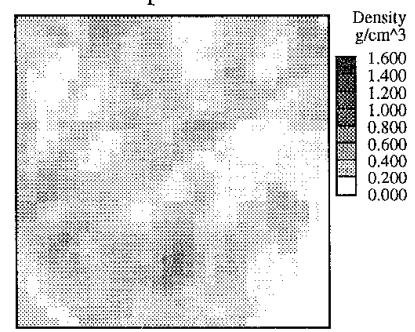

EX: Exp Trial 11

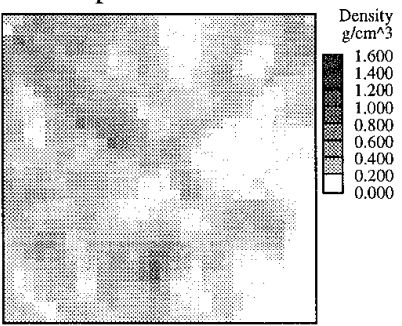

Fig. 3. Reconstructed images of the density distributions shown in Figs. 1 and 2. The reconstruction used a pixel resolution of $2-\mathrm{cm}$ by $2-\mathrm{cm}$, five projection angles, and a projection resolution of $2 \mathrm{~cm}$. The top two images correspond to no explosive present; the bottom two correspond to the presence of a $32-\mathrm{cm}$ by $2-\mathrm{cm}$ block of RDX. 
Varying the threshold and the area of the image above this threshold produces a series of points in the ROC plot. The curves shown in the subsequent figures of this paper correspond to the upper envelope of these points. This upper envelope is essentially the maximum true-positive fraction value for a given false-positive fraction.

\section{RESUlts}

Figure 4 shows the ROC curves for the $8 \times 8 \mathrm{~cm}$ explosive sheet. The number of angles varied from 3 to 5 and the pixel resolutions from $1 \times 1 \mathrm{~cm}$ to $3 \times 3 \mathrm{~cm}$. The projection resolution was $1 \mathrm{~cm}$ for the $1 \times 1 \mathrm{~cm}$ case, and $2 \mathrm{~cm}$ for both the $2 \times 2 \mathrm{~cm}$ and $3 \times 3 \mathrm{~cm}$ cases. The curves show that the reconstruction using 5 projection angles provides essentially a $100 \%$ true positive detection with only a few percent of false positives. The curves also show that increasing the resolution does not significantly change the system performance. This suggests that while higher resolutions have the capability of providing more information, the underdetermined nature of the reconstruction problem does not allow this additional information to be utilized. The data also suggests that it might be possible to relax the projection resolution from $2 \mathrm{~cm}$ to 3 $\mathrm{cm}$ without a significant decrease in system performance.

Figures 5 and 6 show the ROC curves for the rectangular shaped explosive. The reconstruction in Fig. 5 used a $2 \times 2 \mathrm{~cm}$ pixel size and a $2-\mathrm{cm}$ projection resolution. The reconstruction in Fig. 6 used a $1 \times 1 \mathrm{~cm}$ pixel size and a $1-\mathrm{cm}$ projection resolution. For either pixel size the most significant increase in system performance occurs when the number of projection anglesis increased. Comparison of the two figures also shows that there is little improvement in system performance with smaller pixel resolutions. For the rectangular shaped explosive, it appears that at least 11 projection angles will be required to provide a system performance comparable to that obtained using the square shaped explosive and five projection angles.

All of the data in Figs. 4-6 used 25 iterations of the Maximum Likelihood algorithm. Increasing the number of iterations to 50 did not significantly increase system performance.

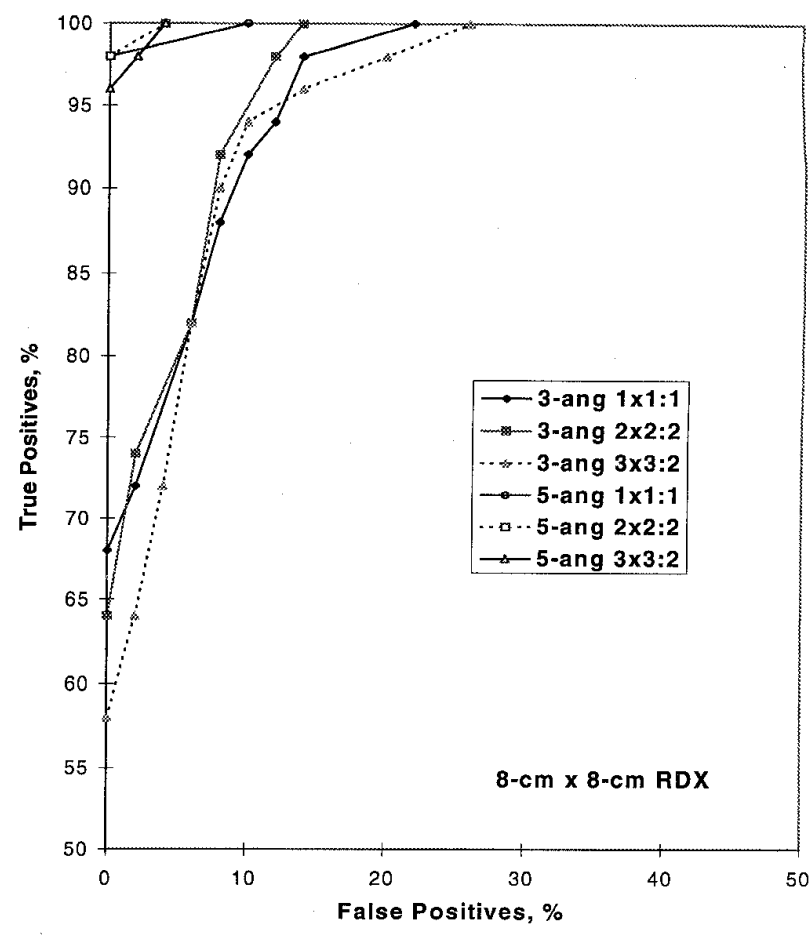

Fig. 4. ROC curves showing the effect of varying the number of projection angles, projection resolution, and the pixel size used in the tomographic reconstruction.

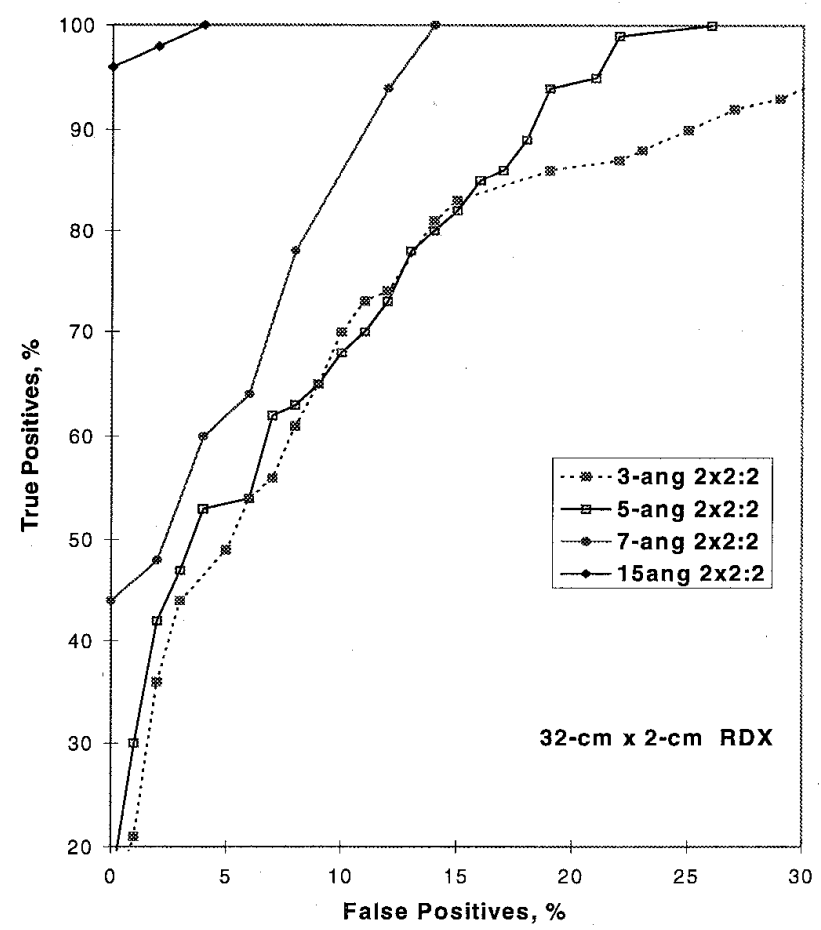

Fig. 5. ROC curves showing the effect of varying the number of projection angles used in the tomographic reconstruction. The reconstruction used a pixel resolution of $2-\mathrm{cm}$ by $2-\mathrm{cm}$ and a projection resolution of $2 \mathrm{~cm}$ 


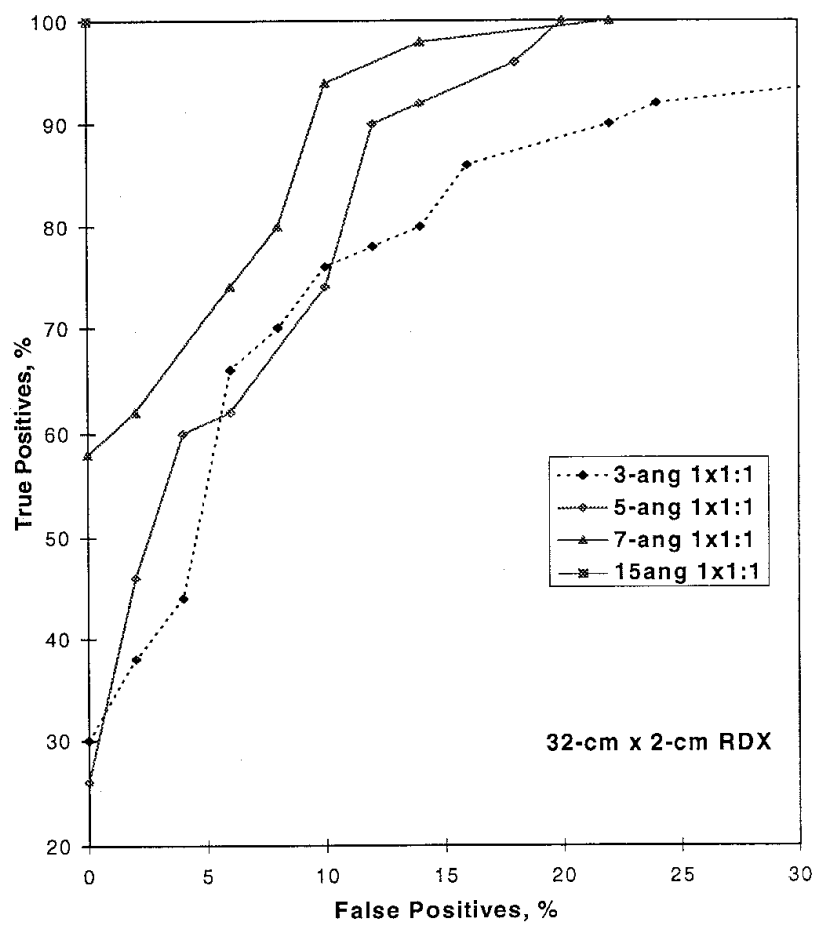

Fig. 6. ROC curves showing the effect of varying the number of projection angles used in the tomographic reconstruction. The reconstruction used a pixel resolution of $1-\mathrm{cm}$ by $1-\mathrm{cm}$ and a projection resolution of $1 \mathrm{~cm}$. Note the single 15 -angle point is located in the upper left-hand corner.

\section{CONCLUSIONS}

The results presented in this paper indicate that square explosives of approximately $8 \times 8 \mathrm{~cm}$ can easily be detected using a minimum of five projection angles. The detection of rectangular sheets will be more difficult and will require a larger number of projections.

Increasing the number of projection angles has the most significant impact on system performance. Increasing the projection and pixel resolution appears to have minimal effect on system performance.

The above conclusions are dependent on the explosive signature algorithm and on the image processing algorithm used in the system evaluation. Since we used relatively unsophisticated algorithms in this study, improving these algorithms should increase system performance beyond that achieved here. However, the relative importance of the number of projection angles, projection resolution, and number of iterations will probably stay the same.

The results also show that ROC curves provide a useful technique in determining the importance of various reconstruction parameters.

\section{ACKNOWLEDGMENTS}

This work was sponsored by the Federal Aviation Administration Technical Center under contract DTFA03-03$\mathrm{X}-0021$

\section{REFERENCES}

[1] T. J. Yule, B. J. Micklich, C. L. Fink, and D. L. Smith, "Illicit Substance Detection Using Fast-Neutron Interrogation Systems," Proc. 4th Int'l Conf. on Applications of Nuclear Techniques, Crete, Greece (June 1994).

[2] C. L. Fink, B. J. Micklich, T. J. Yule, P. Humm, L. Sagalovsky, and M. M. Martin, "Evaluation of Neutron Techniques for Illicit Substance Detection," Nucl. Instr. Meth. B99, 748-752 (1995)

[3] B. J. Micklich, C. L. Fink, and T. J. Yule, "Accelerator Requirements for Fast-Neutron Interrogation of Luggage and Cargo," 1995 Particle Accelerator Conference, Dallas, Texas (May 1995).

[4] J. C. Overley, "Determination of $H, C, N, O$, Content of Bulk Materials from Neutron-Attenuation Measurements," J. Appl. Radiat. Isot. 36, 185-191 (1985); "Element-Sensitive Computed Tomography with Fast Neutrons," Nucl. Instr. meth. Phys. Res. B24/25, 1058-1062 (1987).

[5] B. J. Micklich, M. K. Harper, A. H. Novick, and D. L. Smith, "Illicit Substance Detection Using Fast-Neutron Transmission Spectroscopy," Nucl Instr. Meth. Phys. Res. A353, 646-649 (1994).

[6] B. J. Micklich, C. L. Fink and L. Sagalovsky, "Transport Simulation and Image Reconstruction for Fast-Neutron Detection of Explosives and Narcotics," SPIE 2511, 33-44, European Symposium on Optics for Environmental and Public Safety, Munich, Germany, June 1995.

[7] K.Lange and R. Carson, EM Reconstruction Algorithms for Emission and Transmission Tomography," Jour. of Comp. Assisted Tomography 8(2), 306-316, 1984. 